Utah State University

DigitalCommons@USU

$1-1-1966$

\title{
Simultaneous Comparison of RF Probe Techniques for Determination of Ionospheric Electron Density
}

K. D. Baker

Utah State University

A. M. Despain

Utah State University

J. C. Ulwick

Follow this and additional works at: https://digitalcommons.usu.edu/sdl_pubs

\section{Recommended Citation}

Baker, K. D.; Despain, A. M.; and Ulwick, J. C., "Simultaneous Comparison of RF Probe Techniques for Determination of Ionospheric Electron Density" (1966). Space Dynamics Lab Publications. Paper 5.

https://digitalcommons.usu.edu/sdl_pubs/5

This Article is brought to you for free and open access by the Space Dynamics Lab at DigitalCommons@USU. It has been accepted for inclusion in Space Dynamics Lab Publications by an authorized administrator of DigitalCommons@USU. For more information, please contact digitalcommons@usu.edu.

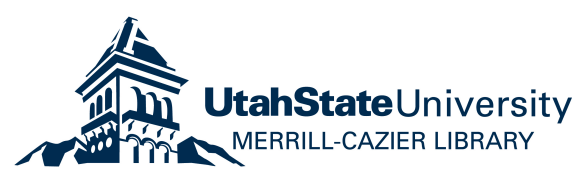




\title{
Simultaneous Comparison of RF Probe Techniques for Determination of Ionospheric Electron Density
}

\author{
K. D. Bakkr and A. M. Despain \\ Upper Air Research Laboratory, University of Utah, Salt Lake City
}

\author{
J. C. ULwick
}

Air Force Cambridge Research Laboratories

L. G. Hanscom Field, Bedford, Massachusetts

\begin{abstract}
Three radio-frequency (RF) probe techniques-standing wave impedance probe, plasma frequency probe, and resonance rectification probe-have been simultaneously flown on rockets for ionospheric measurements. The results of the three probes are compared to establish the relationships among them. The impedance probe and plasma frequency probe measurements are in general agreement with each other and with other independent meas urements. A model of antenna-ionospheric interaction is used that neglects ion sheath and magnetic field effects. The resonance rectification probe shows resonance effects including peaks and minimums that are a function of probe dc bias. The frequency of the dominant resonance peak does not correspond to the plasma frequency but is near a lower frequency of an impedance series resonance as measured by the plasma frequency probe.
\end{abstract}

\section{INTRODUCTION}

The Air Force Cambridge Research Laboratories and the Upper Air Research Laboratory at the University of Utah have jointly developed several types of radio-frequency (RF) probes for measurement of ionospheric electron density. Various experiments have been flown on rockets and satellites providing direct, in situ, measurements. This paper discusses the results of three separate probe techniques that were simultaneously flown on each of several independent vehicles to allow for the evaluation of the various probe techniques and to check the validity of the simple probe theories heretofore presented.

Instrumentation techniques for each $\mathrm{RF}$ probe have been amply discussed in detail in the literature [Haycock and Baker, 1961; Ulwick et al., 1962; Haycock and Baker, 1962; Ulwick et al., 1964; Despain, 1964]; therefore, only brief descriptions will be given here. Each technique utilizes a pair of 2.5- to 3-meter probes, sometimes referred to as a dipole antenna, mounted normal to the longitudinal vehicle axis and extended into the ionospheric plasma. The three systems may share the same antenna through multiplexing techniques, or there may be several sets of antennas operating simultaneously on the same vehicle. In each of the three techniques, the antenna is excited with a low-level $R F$ signal (approximately 0.5 volt $\mathrm{rms}$ ) in the $0.5-$ to $15-\mathrm{Mc} / \mathrm{s}$ range; in addition, a slowly varying bias voltage from 0 to +5 volts is placed on the antennas with respect to the vehicle skin. In each case, an analysis of the effects of the plasma on the antenna $R F$ impedance or on the dc current variations as a function of frequency determines the measured value of plasma frequency, or the corresponding electron density.

\section{RF Probes}

The standing wave impedance probe (SWIP) [Haycock and Baker, 1961; Ulwick et al., 1964] measures the impedance of a dipole antenna being driven by one or two time-multiplexed RF frequencies. The impedance probe frequencies are set so that one or the other is near the local plasma frequency throughout the experiment. The RF fields from the antenna excite motion in the plasma electrons, which in turn modifies the antenna impedance. This change of impedance is indicated by a change in the standing wave pattern of the voltage along an artificial transmission line connecting the driving RF oscillator and the antenna. The voltage standing wave pattern is sampled, de- 
tected, and telemetered to a ground station, where it is recorded for further analysis.

Local electron density is calculated from the departure of the antenna impedance from its free space value, using a simplified analysis. The impedance change is assumed to result solely from a change in the real part of an effective complex permittivity $\epsilon^{\prime}$ of a locally homogeneous ionized gas as expressed in the Appleton-Hartree theory [Ratcliffe, 1962].

$$
\operatorname{Re}\left(\epsilon^{\prime}\right)=\epsilon_{0}\left[1-\frac{\omega_{N}^{2}}{\omega^{2}+\nu^{2}}\right]
$$

where electron thermal motions and the terrestrial magnetic field are ignored, and where

$$
\begin{gathered}
\omega_{N}=2 \pi f_{N}=e \sqrt{N / \epsilon_{0} m} \\
\quad=\text { electron plasma frequency } \\
\omega=2 \pi f=\text { operating frequency. } \\
N=\text { electron density. } \\
\nu=\text { electron collision frequency. } \\
e=\text { electronic charge. } \\
m .=\text { electronic mass. } \\
\epsilon_{0}=\text { free space permittivity. }
\end{gathered}
$$

An equivalent circuit of the antenna based on the above model is shown in Figure 1. The antennas used are always short compared with the operating wavelengths and, hence, have a free space impedance that is essentially a capacitive reactance, of magnitude $X_{0}=1 / \omega C_{0}$. Solving for the plasma frequency $f_{y}$ in terms of the changes $\Delta X$ of the antenna reactance and resistance $\Delta R$ from their free space values gives

$$
f_{N}^{2}=\frac{\Delta X+(\Delta R)^{2} / \Delta X}{X_{0}+\left[\Delta X+\frac{(\Delta R)^{2}}{\Delta X}\right]} f^{2}
$$

or in terms of electron density per $\mathrm{cm}^{\mathrm{s}}$

$$
N=f_{N}{ }^{2} / 80.6 \times 10^{\circ}
$$

Where $v$ is much smaller than $\omega, \Delta R$ becomes negligible, giving

$$
f_{N}^{2}=f^{2} \Delta X /\left(X_{0}+\Delta X\right)
$$

By (2) and (3) the antenna impedance changes, as measured by the standing wave technique, are converted into plasma frequencies or electron densities. The authors are well aware that more sophisticated models of antenna-

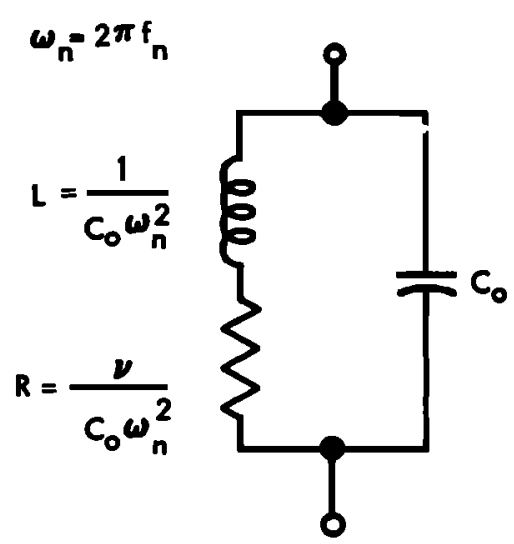

Fig. 1. Simplified equivalent circuit of a short dipole antenna immersed in the ionosphere.

plasma interaction exist [Bramley, 1962; Kaiser, 1962; Balmain, 1964], but part of the purpose of this study was to determine the usefulness of a relatively simple model.

In contrast to the standing wave impedance probe, the plasma frequency probe (PFP) [Haycock and Baker, 1962] excites the antenna with a variable frequency that sweeps from approximately 0.5 to $10 \mathrm{Mc} / \mathrm{s}$. The resonance frequencies of the antenna when immersed in a plasma are sensed by measuring the phase angle between the RF antenna current and voltage as a function of frequency.

Occurrence of a parallel resonance condition associated with the plasma frequency is expected (see Figure 1). Other resonance conditions may also occur, since the equivalent circuit of Figure 1 represents a simplified model which neglects the effects of the terrestrial magnetic field and plasma inhomogeneities, including ion sheaths and electron acoustical waves.

The resonance rectification probe (RRP) [Despain, 1964] operates in conjunction with the plasma frequency probe, using the same antenna and RF sweep system. A dc bias voltage ( 0 to +5 volts), stepped with successive RF oscillator sweeps, is applied with respect to the vehicle body. The resulting dc antenna current is monitored, amplified, telemetered, and recorded along with the phase and frequency information of the plasma frequency probe.

It has been contended that the frequency 
where the dc current reaches a maximum in the resonance rectification probe is the local electron plasma frequency [Takayama, 1960; Miyazaki et al., 1960; Ichikawa and Ikegami, 1962; Hirao and Muraoka, 1964]; however, other investigators do not agree with this conclusion [Harp, 1963; Fejer, 1964; Wimmel, 1964; Gierke et al., 1964]. Thus, one purpose of this study was the investigation of resonance rectification phenomena in general, as well as the comparison of the resonance rectification frequency with values of plasma frequency as measured by the other experiments.

\section{Measurements}

The standing wave, plasma frequency, and resonance rectification probes have been flown together on various aerospace vehicles. In this paper, comparisons are made of the results from each of the probes for some of these flights.

The set of three probes that made up part of the payload of an Aerobee rocket designated AC 3.603 is shown in Figure 2. This rocket was fired into a nighttime aurora and has been described in detail by Ulwick et al. [1965]. The antennas used for each probe were a dipole of two 2.5-meter Fiberglas wrapped whips for the impedance probe, and a dipole of 3-meter telescoping elements for the plasma frequency and resonance probe combination. The telescoping antennas consisted of seven concentric aluminum tubes, the largest having a $0.96-\mathrm{cm}$ radius and the smallest a $0.16-\mathrm{cm}$ radius. The two sets of dipole antennas were mounted so as to be mutually perpendicular to the rocket axis and to each other.

The impedance probe operated alternately at 3 and at $7.2 \mathrm{Mc} / \mathrm{s}$. The plasma frequency and resonance rectification experiments used a 0.5to $10-\mathrm{Mc} / \mathrm{s}$ sweep with 16 discrete bias voltages from 0.0 to 5.0 volts applied in sequence, one fixed voltage for every 0.1 -second sweep period.

Figure 3 shows a section of the telemetry record from this flight, with time increasing to the left. The measurement function of each subcarrier oscillator is labeled. The standing wave impedance probe data were reduced automatically; the other probe data were read manually.

The results from the plasma frequency and the standing wave impedance probes for this flight are shown in Figure 4. The plasma frequency measurements from each probe during rocket ascent are plotted as a function of time after launch. For this figure, the $7.2-\mathrm{Mc} / \mathrm{s}$

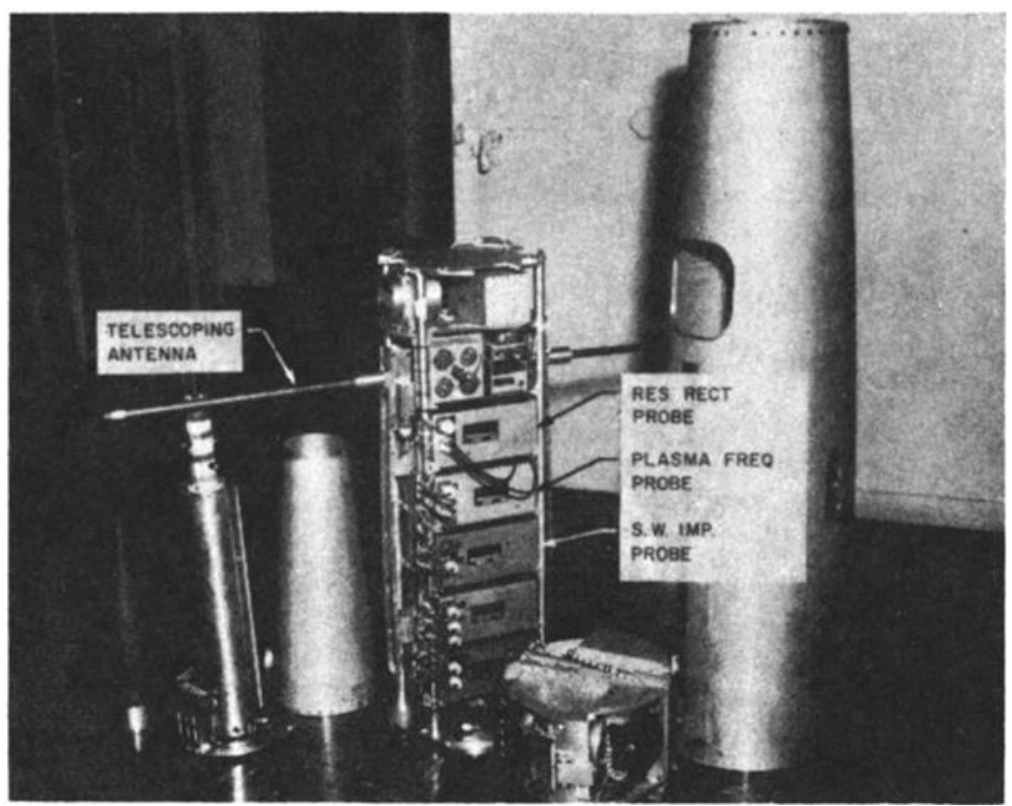

Fig. 2. Aerobee AC 3.603 main payload. 
SWIP results were used since that is well above the electron gyrofrequency (about $1.4 \mathrm{Mc} / \mathrm{s}$ ); however, they are consistent with the $3-\mathrm{Mc} / \mathrm{s}$ results below a plasma frequency of about 2.5 $\mathrm{Mc} / \mathrm{s}$. The results from the two probes are generally in good agreement, particularly around the maximum plasma frequency (4.5 Mc/s), both with each other and with the value of the maximum plasma frequency as determined by the ground-based ionosonde. The maximum discrepancy between the two probe results occurs at the lowest frequency ( $2 \mathrm{Mc} / \mathrm{s}$ ) measured by the plasma frequency probe, where it reads about $15 \%$ higher than the standing wave impedance probe. This discrepancy is removed if the parallel resonant frequency of the plasma frequency probe is assumed to occur not at the plasma frequency, but at the upper hybrid or magnetic plasma resonance frequency given by $f_{z^{2}}=f_{z^{2}}+f_{H^{2}}$, where $f_{z}$ is the electron gyrofrequency. In addition, an onboard measurement of positive-ion densities gives values within
$10 \%$ of the electron densities determined from the plasma frequencies of these probes [Ulwick et $a l ., 1965]$. This is believed to be a good independent check, because, over this altitudinal range, it is reasonable to discount the existence of a significant quantity of negative ions, and charge neutrality requires the positive-ion density to be equal to the electron density.

The three-probe system was incorporated into several instrument capsules that were released from scientific passenger pods attached to ballistic missiles. One of the capsules is shown in Figure 5. The system was basically identical to the one previously described, except that both sets of antennas were of the 3-meter telescoping type.

The data from the plasma frequency probe and the standing wave impedance probe are shown in Figure 6. The agreement between the two methods of plasma frequency measurement is within $10 \%$, the plasma frequency probe again giving higher values at the lower frequen-

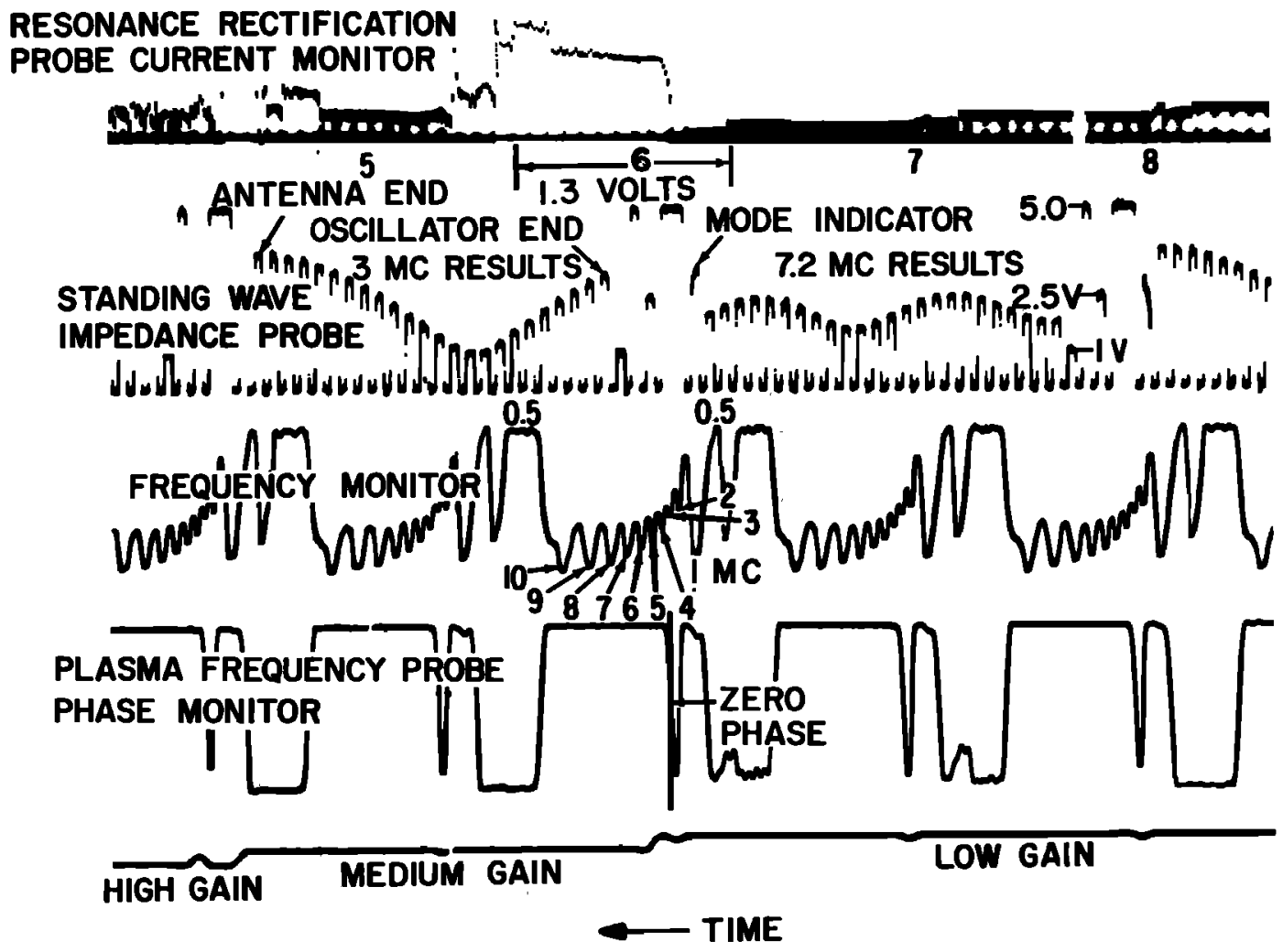

Fig. 3. Aerobee AC 3.603 flight telemetry record. 


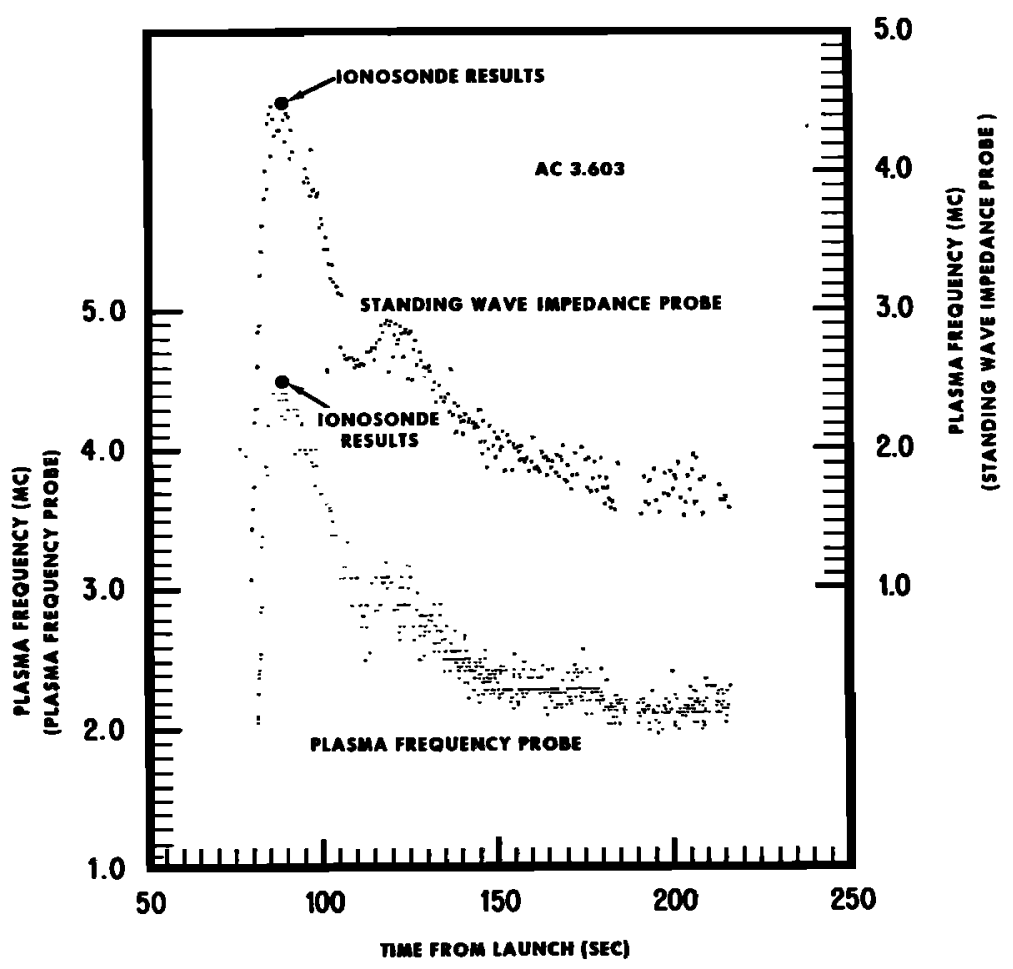

Fig. 4. Comparison of standing wave impedance probe and plasma frequency probe results (AC 3.603).

cies according to the simple analysis used. Of special interest in this case is the indication of a series resonance condition in addition to the usual parallel resonance (plasma frequency) from the plasma frequency probe record.

The flight telemetry record from the threeprobe instrumentation flown on a Black Brant (AC 17.601) research rocket is shown in Figure 7. In this case the impedance probe operated at $3 \mathrm{Mc} / \mathrm{s}$ and was time-multiplexed with the plasma frequency-resonance probe combination using a single dipole antenna (Figure 8). This antenna consisted of two opposed 2.5-meter whips, each of which had a conductor radius of $0.08 \mathrm{~cm}$ and was covered with Fiberglas over the inboard $180 \mathrm{~cm}$ length, leaving the end 70 $\mathrm{cm}$ of the conductor exposed. The sweep frequency range was 0.5 to $5 \mathrm{Mc} / \mathrm{s}$, and the sequential de bias voltages of the probes were $5.2,2.6,1.3$, and 0.0 volts.

The plasma frequency probe phase angle and resonance rectification probe current, each as a function of frequency, are shown in Figure
9. The current is displayed as the lower trace in each of the four sets of curves, corresponding to consecutive de bias voltages. The important feature of these results is the extreme dependence of the observed resonance phenomena on the probe de bias. With a bias of 5.2 volts (Figure 9A) the de current changed very little during the RF sweep period. The next sweep (Figure 9B, 2.6-volt bias) showed a pronounced minimum with no evident maximum. With the bias at 1.3 volt (Figure $9 C$ ), the curve has both a maximum and a minimum, and is typical of the so-called resonance frequency reported by other workers [Hirao and Muraoka, 1964]. Figure $9 D$ shows the largest peak, but no minimum was detectable because the instrumentation was unable to respond to negative currents below $-0.02 \mu \mathrm{amp}$.

The results for the resonance rectification and plasma frequency probes are compared in Figure 10. In contrast to the comparisons made earlier, these are smooth curves rather than raw data-point plots. The rate of data acqui- 


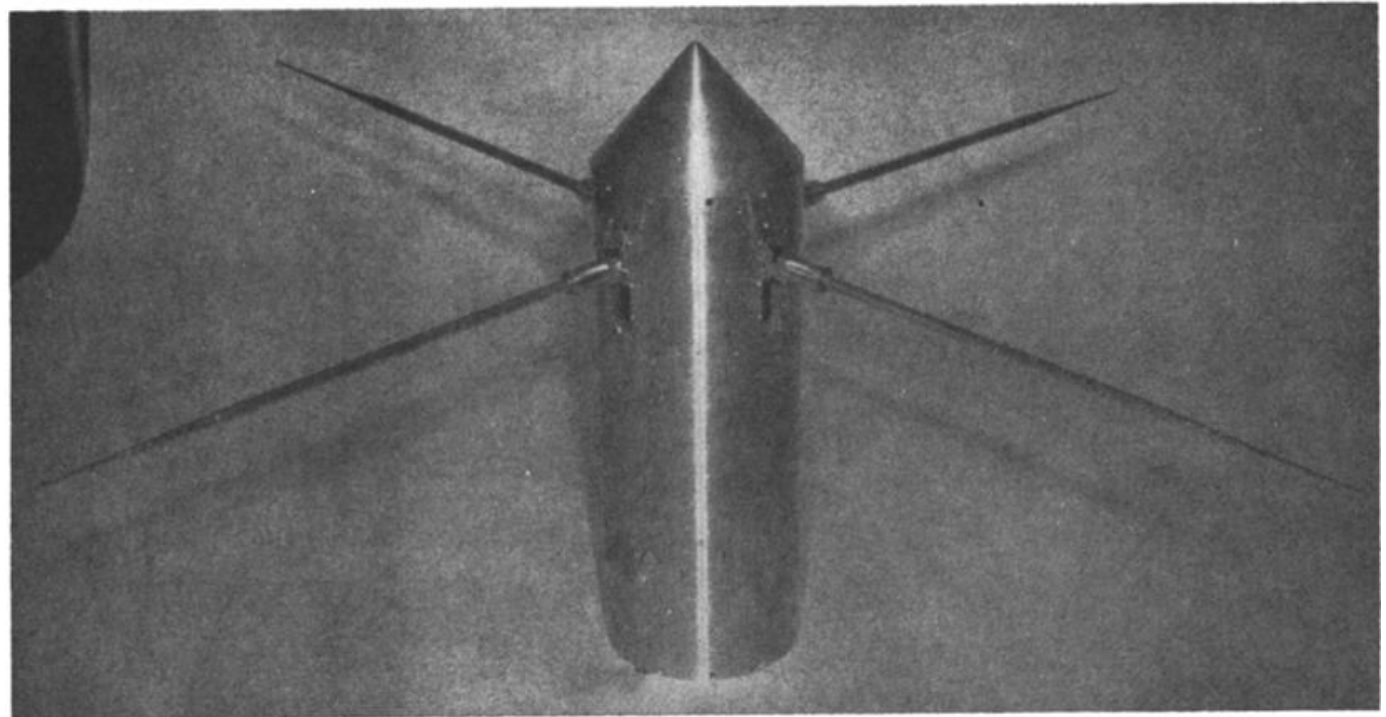

Fig. 5. Pod instrument capsule with telescoping antennas.

sition for this system was quite low compared with the spin rate of the vehicle, and so the smoothing technique improved the visualization of the data correlations. In this flight, as in the latter portions of most rocket and in some satellite flights, a quite noticeable spin modulation of the data occurred that is attributed to a reduction in electron density as the sampling antenna falls back of the vehicle into the rarefied wake region swept out by the vehicle [Haycock et al., 1964]. The resonance data shown are the frequencies corresponding to the de current peaks for the zero-volt bias case. It is clear that this frequency was much lower than the plasma frequency. In fact, the frequency of the dc current peak was near, but slightly lower than, the series resonance frequency. A comparison of the dc current peak and the series resonance frequencies on the other flights, in spite of the poorer technical quality of the resonance data, yields similar results.

The frequency dip in the de current as a function of flight time is shown in Figure 11 for two bias steps. It is noteworthy that the characteristic minimum frequency always occurred between the RF plasma frequencies and the series frequencies. At low bias voltage the current minimum occurred slightly below the plasma frequency, whereas at high bias voltages the minimum occurred slightly above the series frequency.

The parallel resonance mode of the plasma frequency probe was not observed continuously throughout the flight; a notable gap existed above $4 \mathrm{Mc} / \mathrm{s}$. This signal dropout is believed to be due to insufficient instrumentation gain rather than to an ionospheric phenomenon. Ionosonde and standing wave impedance probe results, which are not presented here, show that the plasma frequency for the $E$ region (near the peak in Figure 10) was approximately $4.5 \mathrm{Mc} / \mathrm{s}$. The measurements agree at high densities, but the impedance probe gave unexplained lower values at plasma frequencies below $3 \mathrm{Mc} / \mathrm{s}$.

\section{Discussion of Results}

The plasma frequencies as measured by the plasma frequency probe and the standing wave impedance probe experiments generally agreed between themselves and with other, independent measurements. Measurements of plasma frequency were not greatly affected by the terrestrial magnetic field. The plasma frequencies derived from the plasma frequency probe below $3 \mathrm{Mc} / \mathrm{s}$ were 10 to $15 \%$ lower than those from other methods unless corrected by the equation $f_{X}{ }^{2}=f_{H}{ }^{2}-f_{H}{ }^{2}$. No effect of the applied bias voltage on the measured plasma fre- 
quency was observed. Thus, since this bias voltage modifies the ion sheath surrounding the antenna, it is concluded that the plasma frequency measurements from these probes are not significantly perturbed by the sheath. Since the sheath represents a rather small portion of the total sampling volume for a thin cylindrical antenna, it might be expected to play a rather minor role in the measurements well below the operating frequency. Near the plasma frequency the effective sheath capacitance in series with the high-impedance plasma (see Figure 1) would again be expected to be relatively unimportant. Consequently, both these experiments yield data from which the ionospheric electron density can be secured within $10 \%$ with a simple analysis at probing frequencies above $3 \mathrm{Mc} / \mathrm{s}$.
A series resonance frequency was consistently noted in the RF measurements for all the flights. The ratio of the series frequency to the plasma frequency was found to be approximately 0.6 , the specific value of which is, in general, a function of plasma frequency, experiment geometry, and other plasma conditions such as electron temperature.

The maximums in the dc antenna currents of the resonance rectification probe were selfconsistent but did not occur at the plasma frequency. Thus, it appears that the results of the experiments presented here disagree with the measurements and theory presented by Takayama [1960], Miyazaki et al. [1962], Hirao and Muraoka [1964], and Ichikawa and Ikegami [1962]. In fact, the de current peaks were more closely associated with the series resonance

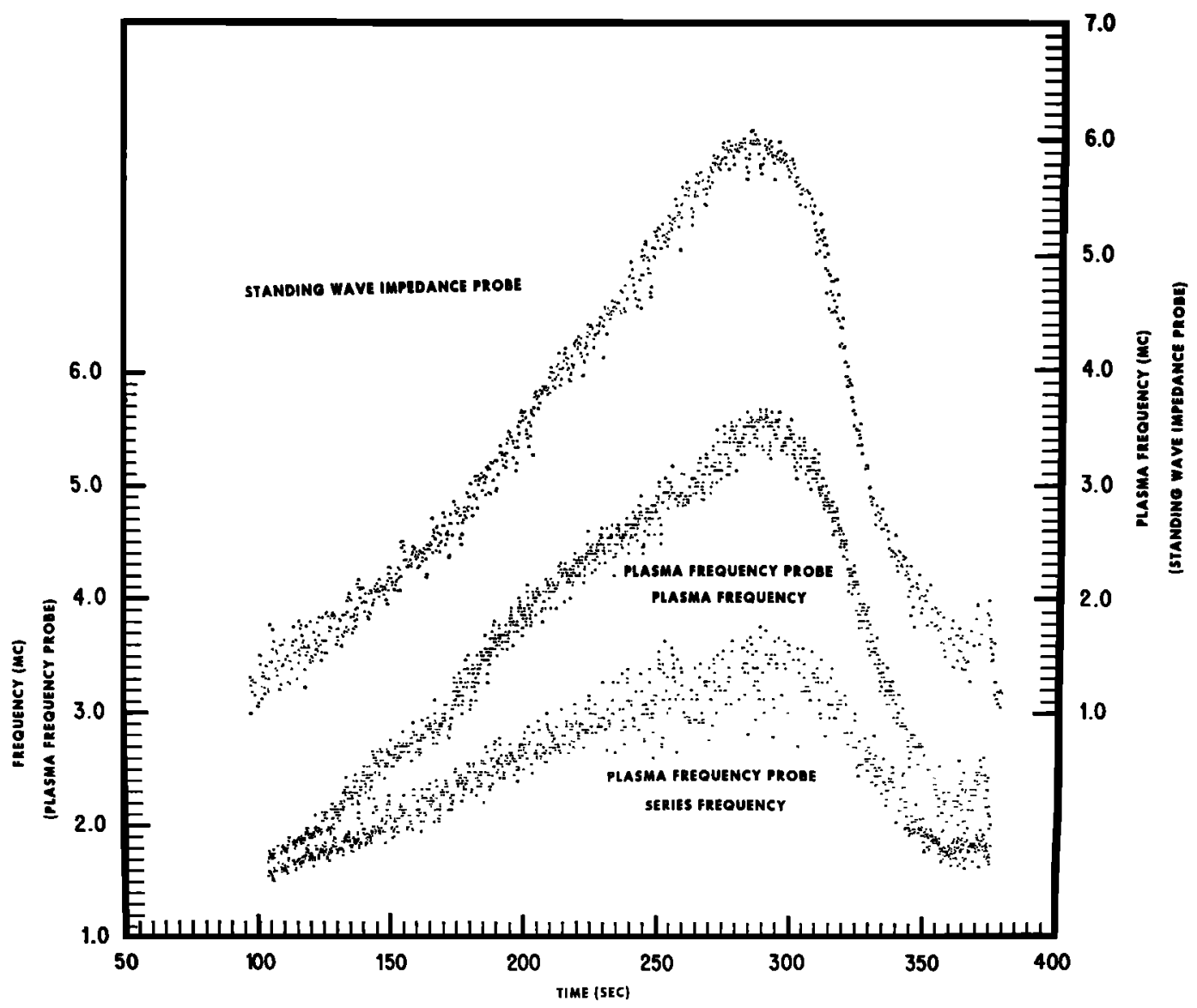

Fig. 6. Comparison of standing wave impedance probe and plasma frequency probe results (pod 1). 


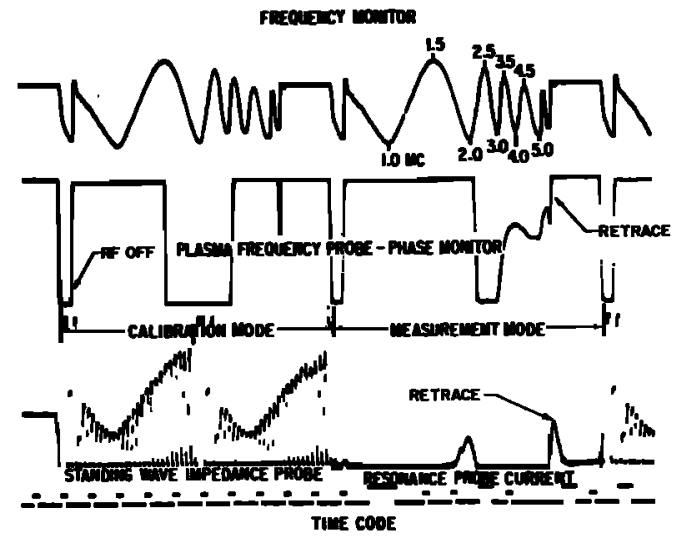

Fig. 7. Black Brant AC 17.601 telemetry record.

frequencies as suggested by Harp [1963], Fejer [1964], Wimmel [1964], and Crawford and Mlodnosky [1964]. Several other de antenna current peaks were occasionally noted on the telemetry records. One of them occurred very near the electron gyrofrequency. Another, observed on the Black Brant flight for the zerovolt bias case, was consistently larger than the peak associated with the series frequency. It increased with increasing rocket altitude to about $3.5 \mathrm{Mc} / \mathrm{s}$, remained essentially constant until rocket apogee, and then vanished. This resonance peak and several less dominant ones do not appear to be related to any other signifi-

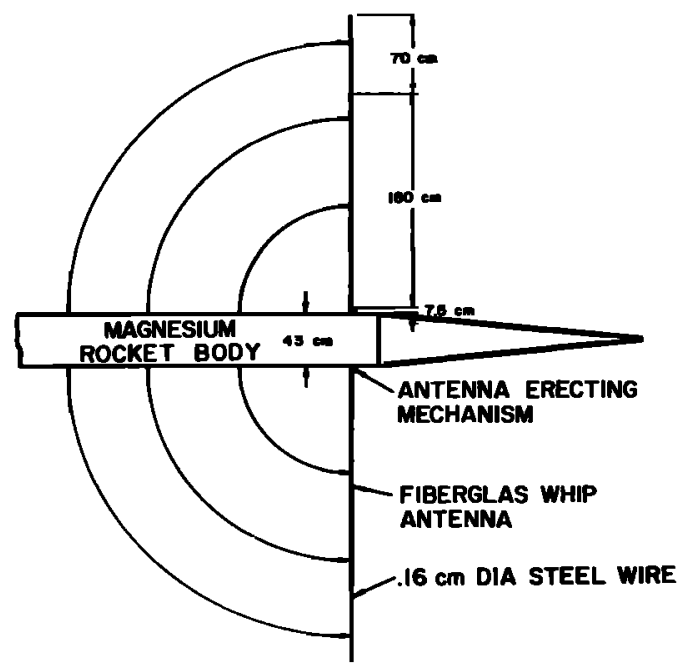

Fig. 8. Black Brant antenna configuration (AC 17.601).
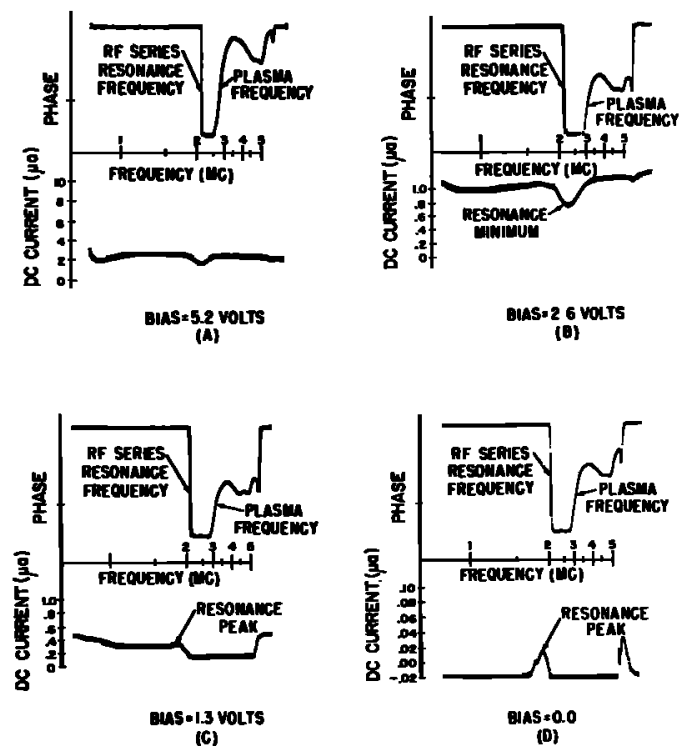

Fig. 9. Examples of resonance phenomena (AC 17.601).

cant frequency. All the resonance phenomena from the plasma frequency and resonance, rectification probes disappeared entirely at low altitudes where the collisional frequency becomes comparable with the plasma frequency.

A dip in the de antenna current versus frequency curve was noted on several of the flights. This minimum was very sensitive to the probe de bias and always occurred at a frequency between the series resonance frequency and the plasma frequency, being displaced toward the plasma frequency as the bias decreased. (These

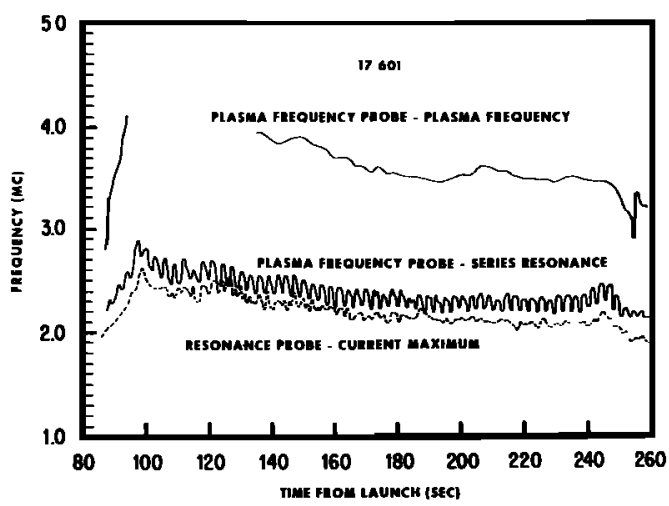

Fig. 10. Comparison of series, plasms, and resonance frequencies (AC 17.601). 


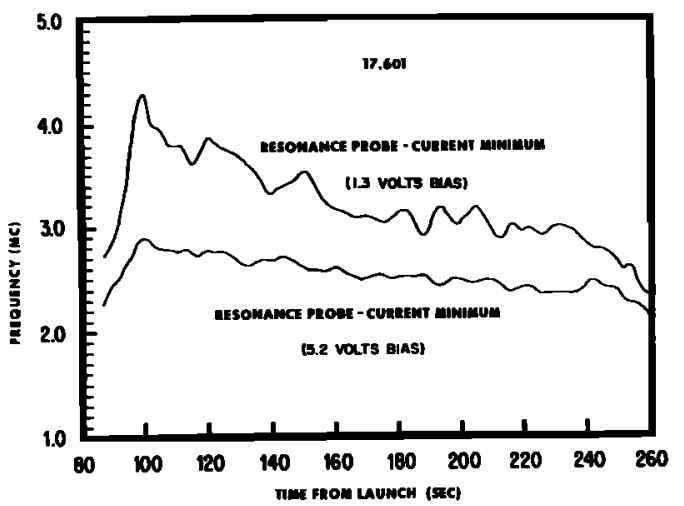

Fig. 11. Resonance rectification probe current Minimum frequencies (AC 17.601).

effects of probe bias are equally true in the regions where the rocket was moving several kilometers during a biasing sequence and near apogee where movement was small.) It would be interesting to observe the resonance phenomena for the region of negative probe currents; this observation is planned for future experiments.

As was mentioned earlier, the resonance rectification probe was operated with two different sizes of electrodes. The small-radius probe $(r \simeq$ $0.08 \mathrm{~cm}$ ) gave results of better quality than the larger one $(r \simeq 0.8 \mathrm{~cm})$. Thus, it appears that these resonance effects are more pronounced if the probe radius is small compared with the Debye length, which does not confirm the conclusions of Crawford and Harp [1965].

\section{Conclusions}

The simple theoretical model neglecting magnetic field and sheath effects used in the analysis of the data from the standing wave impedance and plasma frequency probes was found to be adequate for frequencies of $3 \mathrm{Mc} / \mathrm{s}$ or higher. The data suggest that a more sophisticated theory including the terrestrial magnetic field needs to be invoked as the probing frequencies are reduced below about $3 \mathrm{Mc} / \mathrm{s}$. In contrast to dc-type probes, these two RF probe techniques appear to be relatively independent of the effects of variable sheath dimensions.

Both the standing wave impedance probe and the plasma frequency probe are very useful for ionospheric electron-density measurements. The plasma frequency probe has the advantage of a particularly simple analysis; the resonance rectification probe, on the other hand, is perhaps most useful in studies of plasma characteristics other than electron density.

Acknowledgments. It is a pleasure to acknowledge the support of the following groups: the Aerospace Instrumentation Laboratory of Air Force Cambridge Research Laboratories, for telemetry and vehicle integration support; and the research staffs of Boston College and the University of Utah Upper Air Research Laboratories, for conducting the experiments and analyzing the data.

\section{REFERENCES}

Balmain, K. G., The impedance of a short dipole antenna in a magnetoplasma, IEEE Trens., Antennes and Propagation, AP-15(5), 605-617, 1964.

Bramley, E. N., The impedance of a short cylindrical dipole in the ionosphere, Planetary Space Sci., 9, 445-454, 1962.

Crawford, F. W., and R. S. Harp, The resonance probe-A tool for ionospheric and space research, J. Geophys. Res., 70(3), 587-586, 1965.

Crawford, F. W., and R. F. Mlodnosky, Langmuir probe response to periodic waveforms, $J$. Geophys. Res., 69(13), 2765-2773, 1964.

Despain, A. M., A plasma resonance and electron temperature experiment, Sci. Rept. S, USAF contract AF 19(628)-447, Rept. AFCRL 64-809, University of Utah, 1964.

Fejer, J. A. The interaction of an antenna with a hot plasma and the theory of resonance probes, Radio Sci. (J. Res. NBS), 68D(11), 1171-1176, 1964.

Gierke, G. V., G. Müller, G. Peter, and H. H. Rabben, On the influence of ion sheaths upon the resonance behaviour of a RF plasma probe, Z. Naturforsch., 19a, 1107-1111, 1964.

Harp, R. S., A theory of the resonance probe, M. L. Rept. 1117, Stanford University, Fifth Annual Meeting of the American Physical Union, San Diego, California, November 1963.

Haycock, O. C., and K. D. Baker, Measuring antenna impedance in the ionosphere, Electronics, $\$ 4(2), 88-92,1961$.

Haycock, O. C., and K. D. Baker, New ionosphere measurement technique: Plasma frequency probe, Electronics, $\$ 5(48), 81-83,1962$.

Haycock, O. C., K. D. Baker, and J. C. Ulwick, Experiences with the impedance probe on satellites (presented at IEEE International Convention, New York, March 23-26, 1964), Proc. IEEE, 62(9), 1029-1033, 1964.

Hirao, K., and T. Muraoka, Resonance probe, Cospar Inform. Bull. 17, February 1964.

Ichikawa, Y., and H. Ikegami, Theory of resonance probe, Progr. Theoret. Phys., 28(2), 315, 1962.

Kaiser, T. R., The admittance of an electric dipole 
in a magnetoionic environment, Planetary Space Sci., 9, 639-657, 1962.

Miyazaki, S., K. Hirao, Y. Aono, K. Takayama, H. Ikegami, and T. Ichimiya, Resonance probe -A new probe method for electron density and electron temperature in the ionosphere, Rept. Ionosphere Space Res. Japan, 14(2), 148-159, 1960.

Ratcliffe, J. A., The Magneto-Ionic Theory and Its Applications to the Ionosphere, Cambridge University Press, Cambridge, England, 1962.

Takayama, $K$., Plasma resonance in a radio frequency probe, Phys. Rev. Letters, $5(6), 238-240$, 1960.

Ulwick, J. C., W. Pfister, O. C. Haycock, and K. D. Baker, Description of standing wave im- pedance probe, Cospar Inform. Bull., 17, February 1964.

Ulwick, J. C., W. Pfister, O. C. Haycock, and K. D. Baker, Rocket measurements with electron and ion probes in an aurora, Space Res., 5, 293-311, 1965.

Ulwick, J. C., W. Pfister, R. P. Vancour, R. T. Bettinger, O. C. Haycock, and K. D. Baker, Firing of an Astrobee $\mathbf{2 0 0}$ rocket with a multiple ionospheric experiment, Proc. IRE, 50(2), 22722286, 1962.

Wimmel, $\mathbf{H}$. K., Theory of the plasma resonance probe, Z. Naturforsch., 19a, 1099-1106, 1964.

(Manuscript received May 20, 1965; revised October 8, 1965.) 